Instituto Internacional de Investigación y Desarrollo Tecnológico Educativo INDTEC, C.A.

DOI: https://doi.org/10.29394/Scientific.issn.2542-2987.2021.6.22.15.291-310

OAI-PMH: http://www.indteca.com/ojs/index.php/Revista Scientific/oai

Artículo Original / Original Article

\title{
Intervención Educativa de Estrategias Tecnológicas para Potenciar las Capacidades Investigativas
}

Autor: Luis Manuel Uzcátegui Moreno

Universidad Nacional Abierta, UNA

luismum03@gmail.com

Trujillo, Venezuela

https://orcid.org/0000-0002-0442-3604

\section{Resumen}

El presente artículo trata sobre la intervención relacionada con una situación que involucra un sector de la comunidad universitaria, la cual padece una debilidad en cuanto al uso de habilidades investigativas, en consecuencia, carece de una estrategia que permita su formación. El mismo, tiene como objetivo general, describir una intervención educativa de estrategias tecnológicas para potenciar las capacidades investigativas en los participantes de la Universidad Nacional Abierta (UNA), Centro Local Trujillo, Venezuela. La metodología utilizada se enmarca en un diseño documental caracterizado por uno de campo, orientado desde una perspectiva de estudio de casos. En la captación sobre la información se utiliza las técnicas de observación directa, entrevista no estructurada y para su registro el diario del investigador. La muestra objeto de estudio se conforma por cinco docentes y veinte estudiantes que a su vez son consideradas como sujetos de investigación. Entre los resultados más resaltantes se menciona la formación de colectivos que permite el análisis profundo de la situación problemática con base en su integración, así como la sensibilización de los sujetos sociales acerca de la importancia de implementar las herramientas tecnológicas en el actuar educativo.

Palabras clave: estrategias tecnológicas; capacidades investigativas; intervención educativa.

Código de clasificación internacional: 5802.07 - Formación profesional.

Cómo citar este artículo:

Uzcátegui, L. (2021). Intervención Educativa de Estrategias Tecnológicas para Potenciar las Capacidades Investigativas. Revista Scientific, 6(22), 291-310, e-ISSN: 2542-2987. Recuperado de: https://doi.org/10.29394/Scientific.issn.2542-2987.2021.6.22.15.291-310

Fecha de Recepción: 09-03-2021
Fecha de Aceptación: 28-07-2021
Fecha de Publicación: 05-11-2021 
OAl-PMH: http://www.indteca.com/ojs/index.php/Revista Scientific/oai

Artículo Original / Original Article

\title{
Educational Intervention of Technological Strategies to Enhance Investigative Capacities
}

\begin{abstract}
This article deals with the intervention related to a situation that involves a sector of the university community, which suffers from a weakness in terms of the use of investigative skills, consequently, it lacks a strategy that allows its formation. Its general objective is to describe an educational intervention of technological strategies to enhance the investigative capacities in the participants of the Open National University (UNA), Trujillo Local Center, Venezuela. The methodology used is framed in a documentary design characterized by a field one, oriented from a case study perspective. In capturing the information, the techniques of direct observation, unstructured interview and the researcher's diary are used to record it. The sample under study is made up of five teachers and twenty students who in turn are considered as research subjects. Among the most outstanding results, mention is made of the formation of groups that allows in-depth analysis of the problematic situation based on their integration, as well as the awareness of social subjects about the importance of implementing technological tools in educational activities.
\end{abstract}

Keywords: technological strategies; investigative capacities; educative intervention.

International classification code: 5802.07 - Vocational education and training.

\footnotetext{
How to cite this article:

Uzcátegui, L. (2021). Educational Intervention of Technological Strategies to Enhance Investigative Capacities. Revista Scientific, 6(22), 291-310, e-ISSN: 2542-2987. Recovered from: https://doi.org/10.29394/Scientific.issn.2542-2987.2021.6.22.15.291-310
}

Date Received:

09-03-2021
Date Acceptance:

28-07-2021
Date Publication:

05-11-2021 


\section{Introducción}

Desde hace algunos años, se ha implementado diferentes estrategias pedagógicas a fin de gestionar la construcción del conocimiento promoviendo habilidades sociales e investigativas, que propician la realimentación con el entorno que lo rodea, según Novoa (2013): todo ello, a través de los enfoques actualizados correspondientes a la tecnología. Es necesario implementar estrategias pedagógicas enfocadas en la tecnología, como un medio de interacción con el mundo, facilitando de esta manera un intercambio de ideas que se derivan en una transferencia de saberes y que son aplicadas en distintos ámbitos de desarrollo, tal es el caso de las ciencias educativas, de ingeniería, administración, entre otras.

De esta manera, la integración de estos procesos en los ambientes educativos, permiten entre otras cosas, potenciar en los estudiantes las habilidades investigativas, dando origen a una enseñanza activa y participativa, con el objetivo de promocionar clases innovadoras, que complementen el tradicional sistema de enseñanza educativo. En función de esto, es necesario que el docente asuma el compromiso de ser protagonista de cambio en dicho proceso, de igual forma, hacer del estudiante un ser investigador, creativo, innovador y protagonista de su propia construcción de conocimientos, es decir, el docente tiene como deber aparte de ser el dador de conocimiento, cumplir con la función de orientador, facilitador, guía, mediador y sobre todo ente motivador de los aprendizajes.

Sin embargo, los docentes responsables del área investigativa del Centro Local Trujillo, teniendo conocimiento de estas virtudes, bondades y ventajas ofrecidas por los avances de las nuevas tecnologías, no han establecido de manera cooperativa una política fundamentada en ellas, y orientada al fortalecimiento de las distintas habilidades investigativas que los estudiantes requieren para su completa formación, de modo que es necesario fortalecer sus potencialidades investigativas. Es así como a través del 
diagnóstico y con base en la aplicación de la observación y la entrevista se pudo conocer de esta realidad que conforma la situación problemática.

Con respecto a la importancia, el trabajo de investigación se realiza porque se desea incrementar el nivel de formación investigativa, mediante una intervención educativa y con base en el diseño de una estrategia producto del uso de herramientas tecnológicas. De lo contrario, esta situación afecta de manera importante el progreso en su formación investigativa. Dicha intervención favorece la toma de decisiones con respecto a la realidad social identificada. Desde la perspectiva de la generación de conocimiento, la investigación se orienta desde la corriente de pensamiento del enfoque cualitativo.

En cuanto al objetivo de la presente investigación, se establece describir una intervención educativa de estrategias tecnológicas para potenciar las capacidades investigativas.

\section{EI Problema}

La investigación es una actividad desarrollada por el ser humano consistente a encontrar solución a determinado planteamiento, actividad que obedece a un proceso organizado, sistemático y metodológico emprendido a fin de encontrar esa respuesta en la forma más eficiente posible.

A este respecto, Hernández, Fernández y Baptista (2014a): señala que "la investigación es un conjunto de procesos sistemáticos, críticos y empíricos que se aplican al estudio de un fenómeno o problema" (pág. 4). En consonancia con estos autores se considera importante al momento de encontrar la solución a cierta problemática, llevar a cabo un estudio el cual consista en un procedimiento secuencial, donde el investigador contribuya en virtud de sus experiencias darle a su análisis un enfoque de mayor profundidad.

En tal sentido, su desarrollo permite aportar a la comunidad intervenida 
la generación de nuevos conocimientos. Tal afirmación, confirma su relación estrecha con la transformación del ciudadano, ya que esta es sinónimo de desarrollo, progreso y enriquecimiento intelectual. En esta correspondencia participa la institución universitaria, ambiente donde tiene lugar las actividades concernientes a la formación del estudiante orientadas entre muchas otras, a su uso adecuado, en pro de forjar el talento humano requerido para generar los cambios necesarios en las realidades sociales.

De este modo, las universidades a través de su función docente contribuyen a desarrollar en los estudiantes las distintas habilidades, entre las que cuentan la capacidad para problematizar situaciones, profundizar en el análisis crítico, reflexionar sobre realidades sociales, habilidades de lectura, escritura, comprensión, entre otras, que progresivamente favorezcan su formación académica.

Sin embargo, la realidad existente en el Centro Local Trujillo con respecto a su formación investigativa es distinta, carece de una estrategia robusta orientada a fortalecer las competencias investigativas que requiere el estudiante para responder eficazmente a las demandas generadas de su entorno. Esta percepción fue producto del diagnóstico realizado con base en la herramienta de calidad Matriz FODA, observación directa y aplicación de entrevistas a docentes y estudiantes del Centro Local, en el cual se evidencia además de la carencia de habilidades, una desmotivación entre estudiantes y docentes con respecto a la investigación, así como el poco desarrollo de una praxis investigativa en las asesorías de las diferentes asignaturas. Sin embargo, se divisan fortalezas, oportunidades y amenazas, las cuales han sido objeto de consideración en el desarrollo del proceso indagatorio.

Por otro lado, cabe señalar que la tecnología actualmente provee una variedad de herramientas que muy bien, pueden coadyuvar a la formación investigativa de los actores sociales participantes en la investigación, pero tampoco son del todo tomadas en cuenta, es decir, no existe una política 
colectiva del uso de estas, sino más bien cada docente actúa de manera aislada.

En consecuencia, esta alternativa tecnológica no es aprovechada eficientemente por la comunidad académica como posible fuente que se constituye en oportunidades para proporcionar una alternativa que ayude al desarrollo de las actividades de indagación, desperdiciando de esta manera, la gran posibilidad cierta de incrementar con base en el uso de la tecnología el proceso de su formación académica desde una perspectiva integral.

Es por esta razón, y en virtud de dar paso a la producción de conocimiento desde la óptica del desarrollo de las habilidades investigativas, proceder convenientemente a tratar la situación a partir de una intervención educativa sólida, robusta y en correspondencia con lo plasmado en la Ley Orgánica de Educación (LOE, 2009): donde se enfatiza la interacción académica bajo los conceptos de investigación y, así, generar creatividad e innovación para la transformación educativa.

\section{Referentes teóricos}

\subsection{Intervención educativa}

El profesor está considerado como el mediador entre los estudiantes y el proceso educativo, tiene la finalidad de promover, guiar y facilitar la construcción de sus propios aprendizajes a través del uso de actividades innovadoras, a fin de obtener un crecimiento integral que le permita socializar lo aprendido de manera cooperativa con los demás.

Es importante que tengan desarrolladas las competencias personales, sociales y profesionales, para así, lograr cumplir con las funciones del quehacer educativo, tales como observar, diagnosticar, esbozar, aplicar y evaluar los fenómenos para suscitar dichos aprendizajes, todo ello, por medio de la intervención pedagógica.

Para Touriñán (2011): “la intervención educativa es la acción intencional 
para la realización de acciones que conducen al logro del desarrollo integral del educando" (pág. 283). En este sentido, lo citado es una referencia para opinar que se trata de una actuación ejercida, en este caso por el docente de manera deliberada y expresada mediante la realización de actividades, en virtud de alcanzar las metas propuestas en una situación programada cuyos resultados hacen referencia al logro de esta planificación, que sin duda alguna influye en el progreso del estudiante. De esta manera, el Consejo Nacional de Fomento Educativo (CONAFE, 2012), expresa que:

Las sesiones son el espacio privilegiado para la intervención pedagógica porque se puede crear un clima de confianza que permite a los participantes reflexionar sobre sus prácticas sin temores y con la seguridad de que todo lo que ahí se diga, ahí se quedará, además de que todos pueden aprender de todos (pág. 17).

Por consiguiente, para realizar una intervención es necesario conocer, en forma integral al participante en aquellos aspectos tales como su forma de pensar, de actuar, es importante indagar desde el punto de vista cognoscitivo. Todo esto, se logra si el profesor involucra a las personas que pueden ayudar a perfeccionar la situación problemática tales como orientadores, metodólogos, pedagogos, entre otros; con el propósito de consolidar el proceso educativo. En efecto, se trata de una acción colectiva desarrollada en un ambiente de cooperativismo, donde existan condiciones de armonía que faciliten la mediación, así como la interacción de los grupos interesados.

\subsection{Estrategias tecnológicas}

Las estrategias tecnológicas en el ámbito educativo, surgen como políticas susceptibles de ser adoptadas, cuya finalidad es la transformación de las prácticas llevadas a cabo por docentes y estudiantes y propiciar la adaptación al entorno digital, pero además, se convierte en una herramienta útil que contribuye a innovar distintas maneras para promover el interés en 
potenciar las capacidades investigativas en los participantes.

De esta manera, la tecnología en la educación es considerada como un instrumento importante para afianzar el constructo del conocimiento del estudiante. Al respecto, Fernández (2009), citado por Peña (2018a), indica que:

Las estrategias tecnológicas se entienden como el conjunto de acciones mentales organizadas y sistemáticas que mejoran el rendimiento intelectual durante la solución de problemas y la construcción de saberes" ... "opciones disponibles para construir y mantener una posición tecnológica generadora de ventajas competitivas (pág. 44).

En atención a este apartado, Mora (2015), citado por Guaman (2017): explica que "las tecnologías se integran cuando se usan continuamente para apoyar y promover los objetivos del programa y para involucrar a los estudiantes en el aprendizaje significativo [...]" (pág. 41). Según lo citado, las tecnologías surten un efecto de motivación para que el estudiante se interese por aprender, pero además ese conocimiento aprendido por este medio es internalizado con mayor facilidad e incorporado a su estructura cognitiva a fin de darle significado.

Cabe resaltar, lo planteado en un estudio para la Universidad Nacional Autónoma de Nicaragua por Peña y Pineda (2015), citado por Peña (2018b): quienes exponen, "[...] los docentes en su mayoría poseen lo necesario, lo básico en cuanto al uso de estrategias tecnológicas [...]" (pág. 43). En función de lo citado, para implementar el uso de las nuevas tecnologías en el ámbito educativo es imperioso que los docentes tengan suficiente conocimiento en su aplicación, a manera de facilitar al estudiantado el uso de las Tecnologías de la Información y la Comunicación (TIC).

\subsection{Capacidades investigativas}

El concepto de habilidades investigativas, o capacidades como también 
se le conoce, está considerado a la diligencia del conocimiento que se realiza para dar solución a un fenómeno específico en un lugar determinado, con el apoyo de procedimientos oportunos de la investigación científica, de manera tal que, el óptimo cometido en la obtención de una formación investigativa esté orientada a la acción educativa, donde el papel del profesorado es ser mediador y multiplicador de contenidos tomando en cuenta las habilidades, valores y cualidades de los estudiantes, aunado a esto, se identifiquen sus potencialidades investigativas para dar paso al nuevo conocimiento de manera significativa.

En función de esto, para lograr dichas potencialidades de manera eficiente, es necesario que se avive la formación de habilidades que coadyuven al perfeccionamiento práctico de las actividades investigativas, y así, ayudar al estudiante apropiarse de los debidos conocimientos que todo investigador debe poseer.

Al respecto, Di Virgilio, et al. (2007); López (2006); Gómez (2010); Cuevas, Guillen y Rocha (2011), citados en Rojas y Aguirre (2015): conceptualizan las habilidades como "el pensamiento crítico, la problematización, comunicación oral y escrita, análisis, abstracción, síntesis, entre otras" (pág. 207). El autor hace mención del conocimiento y manipulación de una diversidad de destrezas que el docente debe promover en el estudiante a fin de propiciar en él, ciertas habilidades que requiere poseer y dominar en función de poder dilucidar una situación problemática.

Asimismo, Maldonado, Landazábal, Hernández, Ruíz y Vanegas (2007): menciona que "desarrollar competencias investigativas implica que estas estén relacionadas con el proceso de formación profesional, afianzando habilidades para observar, preguntar, registrar notas de campo, experimentar, interpretar información y escribir acerca de su práctica profesional" (pág. 48). De acuerdo con lo mencionado, las competencias investigativas se manifiestan por medio de conocimientos, habilidades, destrezas, que son 
desarrollados por personas con la intensión de comprender y transformar la realidad en que habita, para ello expresa la necesidad de que se promuevan en correspondencia a su preparación académica una vez que esta inicie, el progreso de estas capacidades favorece al participante o en la persona que la posee potenciar su nivel de investigación.

Es evidente entonces, que en la formación investigativa es conveniente establecer habilidades, que contribuyan al desarrollo de competencias orientadas a facilitar el proceso investigativo, según Barbón, López y Figueredo (2014): está "[...] se fundamenta en reconocer que está integra diferentes saberes cardinales, entre ellos tenemos: conocimientos, habilidades, capacidades, actitudes y valores, los que forman parte del desempeño del profesor universitario [...]" (págs. 254-255).

En el contexto de estas ideas, resulta importante el desempeño de quien se dedica a la formación, este debe preocuparse por inducir al estudiante el deseo e interés por la investigación, instruirlo con esmero, dedicación y sobre todo con convicción sobre la base de principios metodológicos, facilitando su motivación mediante procesos de reflexión, critica, análisis, razonamientos, la idea es prepararlo, que busque a través de su formación investigativa transformar su realidad.

Para cumplimiento de tan importante propósito, es fundamental que el sistema de educación universitaria posibilite la formación de ese estudiante a nivel competitivo y contribuya a su realización personal sobre la base de estos procesos.

\section{Marco metodológico}

Este apartado de la investigación hace referencia al procedimiento metodológico seguido, en el cual se describen los métodos, técnicas, tipo de investigación, diseño, entre otros, identificando y explicitando a manera de proporcionar la información requerida para su realización. Al respecto, el 
trabajo estuvo enmarcado en una investigación documental, de acuerdo con Arias (2012a), consiste en:

Un proceso basado en la búsqueda, recuperación, análisis, crítica e interpretación de datos secundarios, es decir, los obtenidos y registrados por otros investigadores en fuentes documentales: impresas, audiovisuales o electrónicas. Como en toda investigación, el propósito de este diseño es el aporte de nuevos conocimientos (pág. 27).

Lo citado, es una referencia que utilizó el investigador para darle solución a la situación planteada. En este orden, procedió a la revisión de fuentes como libros, material digital, videos entre otras, que contienen información reseñada por otros dedicados a la investigación, cuyo resultado fue la conformación del nuevo conocimiento. Es importante resaltar, lo significativo que resultó la interpretación de esta información para la correcta construcción del nuevo conocimiento. Proceso de revisión cuyo carácter interpretativo, generó una lectura comprensiva sobre una realidad existente, que fue concebida previa al conocimiento por construir.

De igual forma, la investigación tiene características de un diseño de campo, ya que el suministro de información se generó mediante la interacción con la comunidad objeto de intervención. En este sentido Arias (2012b), manifiesta que este tipo de indagación:

Consiste en la recolección de datos directamente de los sujetos investigados, o de la realidad donde ocurren los hechos (datos primarios), sin manipular o controlar variable alguna, es decir, el investigador obtiene la información pero no altera las condiciones existentes. De allí su carácter de investigación no experimental (pág. 31).

Lo anteriormente descrito, refiere la forma como se obtuvo la información en el proceso de desarrollo de la investigación, en este caso fue adquirida por el investigador de manera directa, en el propio ambiente de desenlace. De esta manera, se evidenció en la realización del diagnóstico la 
falta de una estrategia que promueva en los estudiantes el desarrollo de sus potencialidades investigativas.

En cuanto al método de trabajo, este hizo referencia al estudio de casos sobre el cual se analizó la realidad concebida en forma intensiva. Este método buscó ante todo expresar soluciones viables para la resolución del fenómeno tratado en los estudiantes de la Universidad Nacional Abierta (UNA), Centro Local Trujillo, Venezuela. Lo anteriormente descrito se argumenta, conforme a lo expresado por Arias (2012c): quien afirma "[...] se entiende por caso, cualquier objeto que se considera como una totalidad para ser estudiado intensivamente" (pág. 33); desde este enfoque generalmente se busca que el proceso de investigación se centre en la especificidad de un tema, logrando que sus aspectos sean tratados de forma minuciosa.

Desde la perspectiva de la captación de información, la investigación utilizó la observación directa y el diario del investigador como técnica e instrumento respectivamente, y una entrevista de tipo no estructurada. A este respecto, Hernández, Fernández y Baptista (2014b): indican que estos tipos de entrevistas "[...] se fundamentan en una guía general de contenido y el entrevistador posee toda la flexibilidad para manejarla" (pág. 403); lo expresado permite opinar que su desarrollo se concibe a partir de una preselección del tema a tratar, su proceso tiene un carácter flexible y su contenido se estructura de manera abierta.

En consecuencia, el investigador recolectó, analizó y registró la información que luego sistematizó. Debido a la manera en que recolectó la información procedió a un proceso de triangulación con fuente de datos, permitiendo la reflexión sobre la veracidad de estos, además de eliminar el posible sesgo producido, logró incrementar la validez de los resultados. La investigación se llevó a cabo durante el cuarto trimestre del año 2019. 


\subsection{Muestra}

La muestra hace referencia a una agrupación que puede estar constituida por individuos, situaciones o eventos sobre los cuales o entorno a ellos se recolectan datos de información. Con base en lo expuesto, se hace referencia a Hernández, Fernández y Baptista (2014c): quienes aseveran que "[...] la muestra es un subgrupo de la población de interés sobre el cual se recolectarán datos, y que tiene que definirse y delimitarse de antemano con precisión, además de que debe ser representativo de la población" (pág. 173). El proceso de su selección quedo fundamentado en la anterior cita, y consistió en un equipo de informantes constituido por cinco docentes colaboradores en el área de investigación y veinte participantes procedentes de distintas áreas del saber universitario del Centro Local Trujillo, estado Trujillo, Venezuela.

\section{Resultados de la intervención educativa}

En virtud de llegar a generar un resultado deseable en la consecución del proceso de investigación, se describe a continuación el desarrollo de la estrategia tecnológica basada en la aplicación de herramientas producto de la tecnología y que ha sido enfocada desde una perspectiva tratada a través de una intervención pedagógica.

El 15 de febrero del año 2019, siendo la 1:00 pm en el salón "Lesbia de Portes" del Centro Local Trujillo, se llevó a cabo el encuentro de saberes, a cargo de un docente dedicado al área de investigación. Por su parte, el investigador dio la bienvenida a los docentes y estudiantes, dándoles las gracias por asistir y recordándoles la importancia que tiene el mismo en su formación como futuros investigadores.

El docente comienza el encuentro solicitando a la audiencia disponerse en forma de medialuna y pronunciando lo siguiente: las herramientas tecnológicas son una alternativa que permiten facilitar el desempeño del investigador, generando con esto un espacio para la reflexión. Al respecto, un 
estudiante expresa: la efectividad de estas herramientas como apoyo al trabajo de investigación estriba en el conocimiento y correcto uso que se tenga de ellas, pero en estos tiempos no están dadas las condiciones que puedan facilitar su acceso, me refiero a que debemos disponer de un servicio eficiente de internet y un flujo eléctrico continuo, el cual desafortunadamente no es así.

De igual forma, un docente manifiesta lo importante en el desarrollo del evento es tener conocimiento de las bondades de estas herramientas y de la ayuda en cuanto a la facilitación que suelen ofrecer a la comunidad que se inicia en actividades de investigación. De esta manera, el investigador resalta el objetivo del encuentro que es capacitar a los actores sociales en la formación investigativa a partir del conocimiento de este tipo de estrategias.

Es así, como el docente expositor inicia el desarrollo de la temática proyectando diapositivas, que consideran la definición de la gestión del conocimiento como un sistema para administrar la recopilación y análisis de cualquier tipo de conocimiento, explicando su uso conforme al desarrollo de cada etapa, por ejemplo, para recopilación de información mediante bases de datos electrónicas, se exponen las siguientes en la tabla 1.

Tabla 1. Bases de datos electrónicas.

\begin{tabular}{|c|c|l|}
\hline Base de Datos & Año & \multicolumn{1}{c|}{ Descripción } \\
\hline Google Académico & 2004 & $\begin{array}{l}\text { Un servidor que permite la búsqueda de bibliografía } \\
\text { especializada se realiza de manera sencilla y en una } \\
\text { gran numero de disciplinas, así como de fuentes, } \\
\text { tomando en consideración que estas indagaciones las } \\
\text { puede hacer desde un solo sitio. Este servidor se } \\
\text { convierte en un instrumento poderoso de ayuda a la } \\
\text { investigación académica. }\end{array}$ \\
\hline EBSCO & 1984 & $\begin{array}{l}\text { Publishing es un recurso electrónico muy utilizado por } \\
\text { bibliotecas de todo el mundo. Se constituye en el mayor } \\
\text { proveedor a nivel mundial, con una cantidad de } \\
\text { información cuantificada en más de 200 bases de datos } \\
\text { a texto completo y secundario. }\end{array}$ \\
\hline ScienceDirect & 1997 & $\begin{array}{l}\text { Considerada como una plataforma electrónica de fácil } \\
\text { acceso y uso que provee a todo aquel que accede a ella } \\
\text { información completa referida a artículos redactado por } \\
\text { investigadores de alto nivel nacional e internacional. }\end{array}$ \\
\hline
\end{tabular}

Fuente: El Autor (2021). 
OAI-PMH: http://www.indteca.com/ojs/index.php/Revista Scientific/oai

\section{Artículo Original / Original Article}

Seguidamente, el profesor procedió a describir aquellas herramientas tecnológicas que facilitan al investigador, llevar un debido control sobre las fuentes de información que permiten recopilar los datos que serán utilizados en el proceso investigativo como se presentan en la tabla 2.

Tabla 2. Herramientas Tecnológicas.

\begin{tabular}{|c|c|l|}
\hline Base de Datos & Año & \multicolumn{1}{|c|}{ Descripción } \\
\hline EndNote & 2016 & $\begin{array}{l}\text { Funciona como una biblioteca y se armoniza con } \\
\text { cualquier mecanismo móvil, tal es el caso de } \\
\text { computadoras, teléfonos y cualquier otro dispositivo } \\
\text { electrónico demostrando en sus resultados un alto } \\
\text { estándar de calidad. }\end{array}$ \\
\hline Mendeley & 2008 & $\begin{array}{l}\text { Se caracteriza por ser un administrador completamente } \\
\text { gratis y forma parte de una gran red académica, se } \\
\text { presenta como de gran ayuda a todo aquel que la utilice, } \\
\text { puede funcionar como biblioteca particular, facilita al } \\
\text { usuario la utilización de citas en la medida que escribe, } \\
\text { así como almacenar información en cualquier } \\
\text { dispositivo. }\end{array}$ \\
\hline
\end{tabular}

Fuente: El Autor (2021).

Con respecto a la parte del procesamiento de datos en la fase de ejecución y con la idea de coadyuvar a la optimización del trabajo se menciona las siguientes herramientas, expuestas en la tabla 3.

Tabla 3. Herramientas para el Procesamiento de Datos.

\begin{tabular}{|c|c|l|}
\hline Base de Datos & Año & \multicolumn{1}{c|}{ Descripción } \\
\hline MATLAB & 1984 & $\begin{array}{l}\text { Es un tipo de lenguaje científico desarrollado como un } \\
\text { espacio que permite la interacción de quienes lo usan, es } \\
\text { muy versátil, a través de esta herramienta se puede realizar } \\
\text { cálculos, desarrollar algoritmos y aplicaciones. Es de fácil } \\
\text { instalación en software comercial o libre (Windows-Linux). }\end{array}$ \\
\hline MAXQDA & 1989 & $\begin{array}{l}\text { Es un tipo de herramienta a través de la cual se puede } \\
\text { organizar cualquier dato investigado, pero también se } \\
\text { puede codificar e interpretar como crear informes, a la vez } \\
\text { de socializar con otros investigadores. Está diseñado para } \\
\text { Windows y para acceder a este hay que cotizar la licencia. }\end{array}$ \\
\hline Software IBM & 1969 & $\begin{array}{l}\text { Es una herramienta bastante eficiente en la realización de } \\
\text { estudios orientados al enfoque cuantitativo, es demandado } \\
\text { por una gran cantidad de investigadores (estudiantes- } \\
\text { docentes) y profesionales de cualquier ámbito de } \\
\text { conocimiento, para su acceso se requiere del pago de una } \\
\text { licencia. }\end{array}$ \\
\hline
\end{tabular}

Fuente: El Autor (2021). 
De esta manera, el profesor culmina su presentación, interviene el investigador y agradece al docente su exposición, posteriormente apertura el ciclo de preguntas y respuesta, en donde los sujetos inician un proceso de interacción con el expositor apoyados en la técnica instruccional conocida como discusión, buscando generar el intercambio de ideas entre el expositor y los actores sociales de la investigación. Seguidamente, un estudiante interviene y agradece en nombre de sus compañeros la gentil acción que ha demostrado la organización del evento, así como el esmero que ha tenido el expositor con respecto a la facilitación de la información.

En tanto que una profesora reconoce que la aplicación de estas herramientas en cualquier investigación es de gran apoyo, también expresó, no todos tienen acceso a este tipo de información. Finalmente, un estudiante comenta, que precisamente esta debilidad fortalece el trabajo interactivo del grupo, es decir, ante esta situación se generaría un proceso de trasferencia de conocimiento basado en la participación colaborativa.

\section{Conclusiones}

Como derivación de lo anteriormente expuesto, se ha podido resaltar la importancia que reviste la integración de los sujetos sociales participantes en la investigación realizada en el contexto de la Universidad Nacional Abierta (UNA), Centro Local Trujillo, estado Trujillo, Venezuela, la misma fue fundamental para elaborar y ejecutar acciones planificadas por el investigador en la concepción de la intervención educativa, pues la reflexión del problema genero la formación de colectivos que permitió el análisis profundo de la problemática convirtiéndose en colaboradores directos.

Como se ha podido observar, en la intervención se escucharon las voces de los participantes, donde se evidenció la colaboración por cuanto cada uno pudo aportar desde su rol particular y más allá de él. Por ende, esta dinámica de trabajo fue una oportunidad para desarrollar acciones que 
contribuyeron a la optimización de procesos de integración en las estrategias que constituyeron la intervención pedagógica en el ámbito tecnológico.

De esta forma, se hace presente la enseñanza activa con los estudiantes y profesores que disfrutan de los equipos tecnológicos, pero que no los usan por falta de orientación, buena metodología, todo ello, en aras de mejorar el proceso enseñanza aprendizaje de calidad, por medio del compromiso y de aceptar los cambios que da el mundo. En cuanto a los objetivos planteados, se pudieron lograr a través de la intervención pedagógica desarrollada, a continuación, se describen dichos logros:

a). Involucramiento de los actores sociales de la Universidad Nacional Abierta ubicado en la ciudad de Trujillo, estado Trujillo, para el fortalecimiento del trabajo en equipo en la planificación, organización, elaboración, ejecución y evaluación de acciones para el mejoramiento de la calidad educativa.

b). Sensibilización de los sujetos sociales con relación a la importancia que reviste el hecho de implementar una estrategia confeccionada, con base en el uso de herramientas tecnológicas que permitieron promover en el estudiante y docente una actitud que facilitó su actuación educativa.

c). Socializar con el cuerpo de profesores y estudiantes acerca de las diversas estrategias innovadoras que guardan relación con los recursos tecnológicos.

d). Transformación de la percepción de los sujetos sociales en cuantos a los cambios a impulsar para el beneficio del colectivo.

e). Consolidación del proceso de investigación con la participación de los involucrados en conjunto con el investigador.

Finalmente, la realidad planteada al inicio fue objeto para el desarrollo de la descripción correspondiente a la intervención pedagógica diseñada, con 
base en la estrategia tecnológica, construida mediante la integración de las mencionadas herramientas provistas por la tecnología, las cuales se presentaron como alternativas para fortalecer y potenciar la capacidad investigativas en los participantes, razón de ser de la presente investigación, además otro aspecto que se pudo comprobar en su realización fue el conocer para actuar.

\section{Referencias}

Arias, F. (2012a,b,c). El Proyecto de investigación: Introducción a la Metodología Científica. 6a Edición, ISBN: 980-07-8529-9. Caracas, Venezuela: Editorial Episteme, C.A.

Barbón, O., López, C., \& Figueredo, D. (2014). Cinco saberes para la formación de la competencia científico-investigativa con enfoque de profesionalización pedagógica. Revista Cubana de Reumatología, 16(2), 253-258, e-ISSN: 1817-5996. Recuperado de: https://www.redalyc.org/articulo.oa?id=451644524012

CONAFE (2012). La intervención pedagógica en educación inicial.

Promotor educativo. Primera edición. Cuadernos Aprender para Enseñar. México, D.F.: Consejo Nacional de Fomento Educativo.

Guaman, J. (2017). Estrategias tecnológicas educativas y su incidencia en la enseñanza aprendizaje de los estudiantes en el tercer año de la unidad educativa “José Rodríguez Labandera”, cantón Quevedo, provincia de los Ríos”. Ecuador: Universidad Técnica de Babahoyo.

Recuperado de: http://dspace.utb.edu.ec/handle/49000/4993

Hernández, R., Fernández, C., \& Baptista, M. (2014a,b,c). Metodología de la Investigación. Sexta edición, ISBN: 978-1-4562-2396-0. México: McGraw-Hill / Interamericana Editores, S.A. de C.V.

LOE (2009). Ley Orgánica de Educación. Gaceta Oficial N N. 5.929 Extraordinario del 15 de agosto. Venezuela: La Asamblea Nacional. 
Maldonado, L., Landazábal, D., Hernández, J., Ruíz, Y., \& Vanegas, H. (2007). Visibilidad y formación en investigación. Estrategias para el desarrollo de competencias investigativas. Studiositas, 2(2), 43-56, e-ISSN: 1909-0366. Recuperado de:

https://dialnet.unirioja.es/servlet/articulo?codigo=2719652

Novoa, J. (2013). La cuestión integral del siglo XXI. Saber, Ciencia y Libertad, 8(1), 71-83, e-ISSN: 1794-7154. Recuperado de: https://doi.org/10.18041/2382-3240/saber.2013v8n1.1879

Peña, R (2018a,b). Aplicación de estrategias tecnológicas en el proceso de enseñanza aprendizaje en las ciencias sociales. Revista Nicaragüense de Antropología, 2(4), 39-45, e-ISSN: 520-9736. Recuperado de: https://doi.org/10.5377/raices.v2i4.7834

Rojas, C., \& Aguirre, S. (2015). La formación investigativa en la educación superior en América Latina y el Caribe: Una aproximación a su estado del arte. Revista Eleuthera, 12, 197-222, e-ISSN: 2011-4532. Colombia: Universidad de Caldas.

Touriñán, J. (2011). Intervención Educativa, Intervención Pedagógica y Educación: La Mirada Pedagógica. Revista Portuguesa de Pedagogía, 283-307, e-ISSN: 1647-8614. Recuperado de: https://doi.org/10.14195/1647-8614 Extra-2011 23 
Nacido en la ciudad de Valera, estado Trujillo, Venezuela,

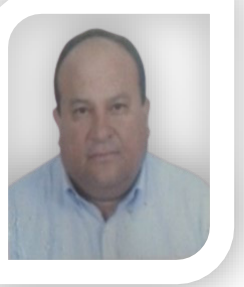

el 3 de febrero del año 1963. Ingeniero Industrial, egresado de la Universidad Nacional Abierta (UNA); e Ingeniero en Construcción Civil graduado de la Universidad Politécnica del Estado Trujillo (UPT); Magíster en Educación Abierta y a Distancia de la Universidad Nacional Abierta (UNA); Diplomado en Gestión de la Investigación, Ciencia y Tecnología de la Universidad de los Andes Núcleo Universitario "Rafael Rangel" (ULA-NURR); Profesor activo y asesor del área de Ingeniería Industrial de la UNA Centro Local Trujillo, Venezuela; Categoría Académica Asistente; he participado en los programas de la dirección de investigaciones y postgrado de la Universidad Nacional Abierta como Tutor Académico en el programa de Maestría de la mencionada Universidad. 